Author Notes:

Abstract Word Count: 299

Manuscript Word Count (main body): 7,658

Number of Tables: 0

Number of Figures: 2

29

*Corresponding Author: 1349 Western Road, London, ON N6G 1H3 Canada:

30 svanhedg@uwo.ca 
Abstract

Natural speech contains many sources of acoustic variability both within and between talkers, which challenges speech recognition in some contexts but may facilitate language understanding in novel listening situations. Despite this ubiquitous variability, most previous studies that have examined the ability to extract sound patterns in speech-known as statistical learning-have used highly controlled, artificial, monotonic streams of syllables. Thus, it is unknown whether variability in speech may help or hinder statistical learning - an important question to resolve if statistical learning does indeed play a role in the segmentation of naturally spoken language, as widely theorized. Here, we assessed whether the use of naturally produced, variable speech sounds produced by multiple talkers benefits or impairs statistical learning, including the ability to generalize patterns to a novel talker. During training, participants listened to approximately 12 minutes of continuous speech made up of repeating trisyllabic words, spoken either by a single talker (single-talker condition) or four talkers speaking for three minutes each (multiple-talker condition). Post-training, all participants completed three assessments of learning: (1) an explicit familiarity rating task, (2) an explicit forced-choice recognition task, and (3) an implicit syllable target detection task. Results indicated that participants in both training conditions showed evidence of statistical learning across all assessments, providing an important demonstration that statistical learning is robust to additional variability in the speech signal. Further, in both the forced-choice recognition task and target detection task, participants in the multiple-talker condition showed evidence of facilitated statistical learning, particularly when listening to a novel talker. In the familiarity rating task, performance was comparable between conditions; however, participants trained with multiple talkers were less likely to conflate word familiarity with talker voice familiarity. Overall, these results suggest that training with multiple talkers can improve aspects of statistical learning across multiple measures of learning.

Keywords: statistical learning, speech segmentation, talker variability, language, memory, auditory perception 


\section{Talker variability facilitates the statistical learning of speech sounds}

Imagine listening to a spoken sentence in your native language and being asked to report how many words you heard. This may seem like an easy task. However, now imagine trying to perform the same task in an unfamiliar language, and the task becomes much more difficult. Speech in an unknown language is perceived as a continuous stream of syllables rather than a sequence of discrete words, and may even feel as if it is being spoken at an unusually rapid pace (Bosker \& Reinisch, 2017). Determining where one word ends and the next word begins in an unfamiliar language is not trivial, as overt acoustic cues such as pauses are not reliable indicators of word boundaries (Cole \& Jakimik, 1980). For beginning learners of a second language, the cues that typically support word segmentation - such as stress patterns and phonotactic regularities - have not yet been learned through experience (cf. Jusczyk, 1999), and may actually be more difficult to learn due to interference from one's native language (e.g., Altenberg, 2005).

This scenario, albeit simplified, highlights the challenges listeners must overcome in trying to parse a continuous speech stream into functionally relevant categories. Yet, the observation that most individuals learn to effortlessly segment speech in their native language over the course of typical development suggests the existence of robust learning mechanisms. One such learning mechanism that is thought to support speech segmentation is statistical learning, which can be defined as the process of becoming sensitive to regularities (patterns) in the environment (Romberg \& Saffran, 2010; Saffran et al., 1996, 1997). Becoming sensitive to patterns allows individuals to learn that certain syllable combinations are likely to co-occur (e.g., ba and $b y$ ), and in turn, that these syllables are more likely to be a part of the same word (i.e., the word "baby"). Syllable combinations that are less likely to co-occur (e.g., ty and ba) are more likely to be indicative of a word boundary (e.g., marking a word boundary in the phrase "pretty baby"). The finding that statistical learning is evident across the lifespan - from eightmonth-old infants (Saffran et al., 1996) to older adults (Schwab et al., 2016) - suggests that statistical learning may support language learning across the lifespan, helping listeners acquire their native language as well as second languages (Ettlinger et al., 2016). In support of this idea, several studies have reported associations between statistical learning measured in the lab and real-world language outcomes (Frost et al., 2020; Lany et al., 2018; Misyak et al., 2010; Misyak \& Christiansen, 2012a; Siegelman, 2020).

Although statistical learning is theorized to play an important role in real world language acquisition, experimental investigations of statistical learning in language often use highly 
artificial, controlled speech sounds that lack the acoustic variability found in real-world listening environments. For example, the seminal study demonstrating statistical learning in eight-montholds by Saffran and colleagues (1996) used speech syllables that were generated from a monotone speech synthesizer (at an average rate of 4.5 syllables per second). The use of speech tokens that are highly similar in nature (i.e., syllables that are generated by a speech synthesizer or recorded individually and then spliced together into a stream) continues to be commonplace in more recent statistical learning studies that have adopted this learning paradigm (Aslin et al., 1998; Batterink, Reber, \& Paller, 2015; Batterink, Reber, Neville, et al., 2015; Batterink \& Paller, 2017; Evans et al., 2009; Hauser et al., 2001; Newport \& Aslin, 2004; Saffran et al., 1997; Toro et al., 2005; Toro \& Trobalón, 2005). While there are several advantages to using controlled speech tokens, such as assuring that other acoustic cues do not unintentionally give listeners diagnostic information from which they could learn, this tight control also reduces ecological validity, raising a question of how well in-lab results generalize to realworld learning situations.

While several studies have used more naturally produced speech sounds - produced by human talkers as opposed to speech synthesizers - these stimuli still tend to be highly controlled and limited in variability. For example, Graf Estes and colleagues used speech tokens recorded by a female talker to investigate whether infants' abilities to segment speech based on statistical cues resulted in enhanced sound-to-meaning mappings (Graf Estes et al., 2007). Similarly, Johnson and Jusczyk (2001), as well as Misyak and Christiansen (2012), used syllable recordings from a female talker to replicate and expand upon the findings of Saffran et al. (1996). Although the source of the speaker was a natural talker in all of these studies, the speech utterances were carefully monitored to be comparable in speaking rate, fundamental frequency, and amplitude to eliminate potential covarying acoustic cues that could assist segmentation. Additionally, in some studies (e.g., Johnson \& Jusczyk, 2001; Batterink \& Paller, 2019), naturally-produced speech tokens were recorded individually and then spliced together to form longer sequences, meaning every time a listener heard a specific sound (e.g., "ba") they would hear the exact same waveform. Again, this approach provides excellent acoustic control of the auditory sequences but does not capture the variability inherent in natural speech (cf. Peterson \& Barney, 1952). A small number of studies have gone one step further, using naturally produced, full sentences to examine statistical learning (Pelucchi et al., 2009; Kittleson et al., 2010; Plante et al., 2015). Importantly, English-learning infants have been shown to successfully extract statistical structure from fluent infant-directed running Italian speech (Pelucchi et al., 2009) and English-speaking adults were found to segment words from natural, 
running Norwegian speech (Kittleson et al., 2010; Plante et al., 2015). Nonetheless, the stimulus streams in these studies are still produced by only a single talker, and do not address the potential importance of inter-talker variability in learning. Overall, then, these studies have demonstrated that listeners can learn statistical structure from real (as opposed to synthesized) speech, though such stimuli remain tightly controlled in many experimental contexts. The potential importance of the "naturalness" of the speech signal is highlighted by a recent metaanalysis of statistical learning studies (Black \& Bergmann, 2017), which found that artificial speech results in reliable learning effects among infants, whereas naturally produced speech does not.

Despite these hints that variability in speech may considerably impact learning outcomes, virtually no studies (of which we are aware) have directly tested the impact of intertalker variability (i.e., exposure to multiple talkers) on the statistical learning of language, particularly with respect to generalization of learning. Answering this question is critical for more fully understanding how statistical learning may operate in real-world contexts, as outside of constrained laboratory settings we receive speech input from a myriad of talkers. Indeed, indexical information (e.g., the identity of who is speaking) is generally known to influence how listeners perceive, remember, and ultimately understand speech. According to theories of talker normalization, listeners must calibrate or "normalize" incoming speech sounds based on vocal characteristics in service of recognition (e.g., Magnuson \& Nusbaum, 2007; Magnuson, Yamada, \& Nusbaum, 1994; Mullennix, Pisoni, \& Martin, 1989; Nusbaum \& Magnuson, 1997; Wong, Nusbaum, \& Small, 2004). There is also significant evidence that talker-specific learning can improve speech understanding, particularly in degraded or noisy listening environments (e.g., Holmes, Domingo, \& Johnsrude, 2018; Johnsrude et al., 2013; Nygaard \& Pisoni, 1998). These results, which highlight the importance of considering talker-voice information in speech processing, suggest that talker-specific effects may be part and parcel of speech recognition. In the context of speech learning paradigms, this suggests that training on one talker may not necessarily fully generalize to a novel, untrained talker.

How might we expect the training of multiple talkers to influence the statistical learning of speech? Perhaps the most straightforward prediction is that increased variability during training would lead to impaired performance, as the diagnostic cues for learning might be harder to extract when experienced over several acoustically distinct speech tokens. Indeed, this pattern of results is generally supported in the perceptual learning literature (e.g., Amitay, Hawkey, \& Moore, 2005), at least from visual and auditory (non-speech) paradigms that typically present 
highly similar perceptual tokens across hundreds, if not thousands, of trials (Karni \& Sagi, 1991; Wright et al., 1997). For example, Karni and Sagi (1991) found robust long-term learning of visual texture patterns, yet learning was highly specific to training (as it did not show interocular transfer and was retinotopically constrained). In audition, Wright et al. (1997) found that individuals can learn to differentiate a 100-millisecond interval from longer intervals with extensive training, yet this interval training does not generalize to different interval durations (e.g., 50- or 200-milliseconds). These findings suggest that training improves perceptual processing in a highly specific way - only benefiting stimuli that closely resemble those encountered in training. Thus, under this view, we would expect optimal statistical learning performance when the training and testing environments are perceptually identical (e.g., when both use the same auditory tokens produced by the same talker).

An alternative prediction is that training with multiple talkers might facilitate some aspects of learning, particularly with respect to generalization and abstraction (cf. Posner \& Keele, 1968). In support of this view, variability during training has been shown to facilitate the generalization of speech categories, particularly when the variability is introduced along a dimension that is irrelevant to the learning task (e.g., increasing talker variability in a phonetic contrast learning task). For example, in a dialect learning task, Clopper and Pisoni (2004) found that talker variability in training facilitated generalization to novel talkers, though initial performance in training was diminished when multiple talkers were heard. This means that although learning may initially appear slower with increased talker variability, benefits are more readily observed when generalizing to novel listening situations. This pattern of findings has also emerged from phonetic contrast training studies. Lively and colleagues found that training with five talkers (versus one talker) improved Japanese listeners' abilities to distinguish the /r/ /I/ contrast for a novel, untrained talker (Lively et al., 1993). These results are consistent with recent theoretical accounts of speech perception, in which listeners are proposed to generalize to the similar (Kleinschmidt \& Jaeger, 2015), meaning increased experience with similar (yet distinct) listening situations might facilitate the learning of the similarities across talkers and promote generalization to novel contexts. Taken together, these results suggest that training on multiple talkers may facilitate learning of speech patterns, particular in situations where listeners must generalize to acoustically novel sounds not experienced as a part of training.

Finally, it is also plausible that training with multiple talkers will not meaningfully influence statistical learning performance. This is because statistical learning differs from perceptual learning paradigms in the sense that the focus of statistical learning is on more high- 
level, abstract word-like units, or transitional probabilities between syllables, rather than on the perceptual features of the signal. For example, learning that the syllable "da" always follows the syllable "bi" might not depend on the specific ways in which these syllables are produced, as English-speaking listeners already possess rich category structures for these sounds. In contrast, learning a difficult, non-native phonetic contrast (e.g., /r/ versus /// for native Japanese speakers) requires the formation of novel categories that may initially overlap in perceptual space with existing category structures. Thus, while it is clear why the perceptual training environment should influence the learning of novel perceptual categories (e.g., Lively et al., 1993), it is possible that the units of learning (i.e., word units, or the transitional probabilities between syllables) in a statistical learning paradigm are sufficiently abstract to not be significantly influenced by the perceptual environment during training.

The present experiments were therefore designed to assess whether training on multiple talkers - as opposed to a single talker - impacts the statistical learning of speech. In both training conditions, participants experienced intra-talker variability, as we recorded multiple tokens of each sound. However, participants in the multiple-talker condition experienced four talkers during training whereas the single-talker participants experienced just one. We adopted the canonical training paradigm of Saffran et al. (1996), in which participants heard a stream of spoken syllables with an underlying transitional structure, and we additionally assessed learning using three separate tasks. Two of these tasks - hereafter referred to as the Familiarity Rating Task and the Forced-Choice Recognition Task - required participants to make explicit judgments about the statistical structure of the speech heard in training. The third task hereafter referred to as the Target Detection Task - was based on response times to individual syllables and can be considered a more implicit measure of learning (Batterink, Reber, Neville, et al., 2015). These three assessments were administered to participants using both a novel talker and a trained talker. This paradigm allowed us to test two hypotheses. The first hypothesis was that relative to participants trained on a single talker, participants trained on multiple talkers should show stronger learning when the assessments used a novel, untrained talker, reflecting enhanced generalization. The second hypothesis was that participants trained on a single talker might show better learning on assessments using the same trained talker, reflecting a benefit of additional experience within the same acoustic environment. 


\section{Method}

\section{Participants}

We recruited 100 participants from Amazon Mechanical Turk $(M=41.9$ years old, $S D=$ 13.1, range of 19 to 71 years old). We recruited participants through CloudResearch (Litman et al., 2017) and restricted recruitment to participants who had passed internal attention checks administered through CloudResearch. Additionally, participants needed to have completed a minimum of 50 prior tasks with at least a $90 \%$ approval rating. The research protocol was approved by the Western Non-Medical Research Ethics Board.

\section{Materials}

The artificial language was based on Saffran et al. (1996). The language consisted of 12 syllables, combined to create four trisyllabic pseudo-words hereafter referred to as words (bidaku, padoti, golabu, and tupiro). All stimuli from the artificial language were recorded by real talkers, as opposed to computer synthesized speech as is often done (e.g., Saffran et al., 1996; Batterink, Reber, Neville, et al., 2015; Toro et al., 2005). Each talker recorded the 12 syllables in isolation four times each, for a total of 48 syllable recordings per talker. Four versions of each syllable were created to increase intra-talker variability and simulate a more naturalistic listening environment in the experiment. These syllables were subsequently randomly assigned during the creation of the sound files (e.g., if the current syllable in the sequence was "pa," then our script would randomly assign one of the four versions of that talker's "pa" to be played), and each participant received a unique ordering of the syllables. During syllable recording, the individual syllable order was randomized, and talkers were guided by a computer script which provided a metronomic countdown prior to each recording. This helped ensure that the syllables were not spoken too quickly or too slowly. Talkers were not made aware of the way in which the syllables would be combined to form the artificial language, in part to discourage the use of any other covarying cues (e.g., unintentionally stressing the first syllable of a word). Recordings took place in a sound-attenuating booth. Six talkers (three female and three male) were recorded for use in training and the assessments. All recordings were digitized at $44.1 \mathrm{kHz}$ with a 16 -bit depth.

Each talker was assigned an image of a headshot and a short, fictional biography. The headshots were not taken from the recorded talkers. Rather, the headshots were selected from an artificial intelligence project (https://generated.photos/) by the authors. The fictional 
biographies written for each talker included (1) a name, (2) hometown and/or current place of residence, (3) profession, and (4) hobbies. The headshot and biographies were implemented to emphasize to participants that the speech recordings were produced by different individual talkers.

\section{Procedure}

The experiment was programmed using jsPsych 6 (de Leeuw, 2015). All participants provided consent by clicking on a computer checkbox acknowledging that they had read the study details and agreed to participate. After providing consent, both participant groups completed the main experiment task (Training Task, Familiarity Judgment Task, Forced-Choice Recognition Task, and Target Detection Task). Figure 1 provides a depiction of the experimental procedure.

[[Figure 1 about here]]

\section{Training Task}

The training task (Fig. 1A) was meant to familiarize participants with the artificial language. There were four blocks in total, with each block containing 180 words (540 syllables). Given the syllable presentation rate of $350 \mathrm{~ms}$, each block thus contained $189 \mathrm{~s}$ of spoken syllables. Within a block, words were represented equally (i.e., bidaku, padoti, golabu, and tupiro were each presented 45 times), and word order was pseudo-random (with a constraint that there could be no consecutive presentations of words).

In the single-talker condition, participants received four blocks of the same talker, randomly selected from one of the six recorded talkers. In the multiple-talker condition, four talkers were randomly selected from the six recorded talkers, with participants receiving a different talker for each block. Prior to beginning each block, participants were shown the headshot and short biography of the talker they would hear. In the single-talker condition, participants were shown the same headshot and biography four times.

Although exposure to artificial languages in statistical learning research is typically passive, requiring participants to simply listen to the sounds with no overt response, we implemented a simple attention check to ensure that participants were indeed listening to the sounds. This was primarily done because the online testing environment prevented us from directly monitoring participant attention and compliance, as would be done in a more traditional laboratory testing environment. Once per block (either one-third or two-thirds of the way into the 
block), the speech stream would pause for up to ten seconds. Participants were instructed to press any key as quickly as possible when this happened to un-pause the speech. If no response was recorded in the allotted timeframe, the attention check was marked as a timeout and the script moved on automatically. Participants were allowed to "time out" once; if participants timed out more than once, they were discarded from the analyses. trained first) was randomized across participants but held constant within a given participant. For participants in the multiple-talker condition, the trained talker was always the final presented talker (i.e., from the fourth block of training), in order to equate the lag time between training and test across the single- and multiple-talker conditions. The novel talker was randomly selected among the remaining pool of recorded talkers (i.e., among the five talkers not selected for training in the single-talker condition and among the two talkers not selected for training in the multiple-talker condition). Prior to beginning each task, participants were explicitly told which talker they would be hearing (receiving the headshot and biography associated with the trained and novel talkers). Participants were explicitly instructed that the novel talker would be speaking the same "language" as the talkers heard in training. The three learning assessments are reported in detail in the following sections.

\section{Familiarity Rating Task} see Batterink et al., 2017). On each trial participants heard a trisyllabic sound and had to judge how familiar it sounded based on their experience in the Training Task. The instructions emphasized that participants should base their judgments on the familiarity of the "words" (i.e., syllable collections), not the familiarity of the talker. There were 12 trials for each talker - four trials contained words (two of two expected syllable transitions, e.g., golabu), four trials contained part-words (one of two expected syllable transitions, e.g., golati), and four trials contained non-words (zero of two expected syllable transitions, e.g., godapi). Trials were randomized, and participants made their responses on a four-point scale (1: not familiar, 4: very familiar). Learning was operationalized in terms of familiarity ratings across each word category 
(word, part-word, non-word). Specifically, we predicted that participants should rate words as more familiar than non-words, with part-words falling between words and non-words.

\section{Forced-Choice Recognition Task} Recognition Task (Fig. 1C). In this task, participants heard two trisyllabic sounds on each trial:

\section{Target Detection Task}

The final learning assessment completed by participants was the Target Detection Task (Fig 1D). This task requires participants to make speeded responses to target syllables in shortened streams of the same artificial language used in the initial Training Task. For each stream, participants were given a target syllable (e.g., ba). This target syllable was printed on the screen, and participants also heard an example of the syllable prior to the initiation of the trial. Participants were instructed to listen carefully for this target syllable and to press the spacebar as quickly and accurately as possible whenever the target syllable was played. Each stream contained 16 words (48 total syllables), and thus contained 4 targets in total. The syllable presentation rate was identical to training $(350 \mathrm{~ms})$, meaning each stream was $16.8 \mathrm{~s}$ in duration. Each word was represented exactly four times, yielding four target syllables per stream. Within each stream, the ordering of the words was pseudo-random and constrained by two primary factors. First, the word containing the target syllable for that trial could not be in the first or final word position (e.g., if the target was bu, the word golabu could not occur in the first or final position). Second, consistent with the longer speech streams heard in training, there were no consecutive repeats of words (e.g., golabu would have to be separated by at least one trisyllabic word before repeating). Participants completed 24 streams for each talker (two trials 
for each of the 12 syllables from the artificial language), yielding 32 targets within each condition bin (syllable position 1, 2, or 3, within a given talker). The ordering of target syllables was randomized within each talker block. Statistical learning was operationalized in terms of response time differences between syllables in the final position of a word (as opposed to syllables in the initial or middle position). Specifically, based on previous work (e.g., Batterink et al., 2015, 2017), we predicted that participants' responses should be fastest for syllables in the final position (as the first two syllables of the word should serve as predictive cues).

\section{Data Exclusion and Analysis}

Participants were removed if they did not perform satisfactorily on the attention check during training (i.e., more than one timeout), as we could not be confident that participants were listening to the stream as intended. This consideration removed five participants. We additionally removed 18 participants for failing to respond to at least $50 \%$ of the target syllables in the Target Detection Task and/or failing to achieve a d' of 1. This was implemented because prior administrations of the Target Detection Task have shown that accuracy is generally high (>80\%; Batterink et al., 2017). Although most participants in the present experiment achieved high accuracy levels, hit rates appeared to be bimodal (Figure S1), with a 50\% threshold conservatively differentiating the two distributions. As such, low accuracy in the present experiment was taken as evidence of task misunderstanding or noncompliance. The final sample size was thus 78 (single-talker: $n=35$, multiple-talker: $n=43$ )

All analyses used mixed-effect models (Baayen et al., 2008) and were performed in $R$ version 3.5.1. Despite the use of three independent learning assessments, there were several properties that were common across tasks. All analyses controlled for test order (novel versus trained talker first) and included random intercepts for test order (with participants nested inside). For each assessment, we first examined evidence of statistical learning using simplified models. Then, if evidence of statistical learning was found for the assessment, we expanded the model to address our primary question of whether training condition (single- versus multipletalker) would influence learning. Each expanded model tested for the main effects of condition and talker, as well as the interaction of condition and talker.

The Familiarity Rating Task, in which ratings were provided on a four-point scale, used a mixed-effects ordinal regression model ("clmm" in R, see Christensen, 2019), with word category (word, part-word, non-word) being treated as an ordered factor. The initial model only contained the word category term and test order; the expanded model added talker and condition. The Forced-Choice Recognition Task, in which performance was binary (correct, 
incorrect) used a generalized linear mixed-effect model ("glmer" in R, see Bates et al., 2020). Additionally, to assess if performance was above chance, we first used one-sample t-tests against the chance estimate of $50 \%$. The primary mixed-effects model included talker and condition, as well as order of the correct answer (first position, second position) and answer confidence as an ordered factor. The Target Detection Task, which employed a continuous measure (response time), used a mixed-effects linear model ("Imer" in R, see Bates et al., 2020), with syllable position (first, second, third) being treated as an ordered factor. For the Target Detection models, we expanded the random effects structure to account for participant slopes for sequence position (i.e., the position of the target within the 48-syllable sequence). The reason we controlled for sequence position was because recent research has suggested that sequence position might be an alternative explanation for syllable position effects (Himberger et al., 2019). The initial model contained syllable position, test order, and sequence position. The expanded model included talker and condition. Similar to prior research that has used the Target Detection Task (e.g., Batterink \& Paller, 2017), participants had to respond within $1200 \mathrm{~ms}$ for their response to be registered as a hit and included in the analysis.

For the Target Detection Task, we additionally used signal detection theory (Macmillan \& Creelman, 2005) to calculate participants' sensitivity ( $\left.d^{\prime}\right)$ to the target syllables. A hit was defined as a keypress within $1200 \mathrm{~ms}$ of a target syllable, a false alarm was defined as a keypress outside of a $1200 \mathrm{~ms}$ window from a target syllable, a miss was defined as a failure to respond to a target syllable within $1200 \mathrm{~ms}$, and a correct rejection was defined as withholding a keypress for nontarget syllables within $1200 \mathrm{~ms}$. In addition to being used as a data exclusion criterion, d' was calculated to assess whether training with a single versus multiple talkers influenced participant sensitivity to target syllables. We calculated $d$ ' using the "psycho" package in $\mathrm{R}$. The resulting d'values were analyzed using a 2 × 2 ANOVA, with condition (single-, multiple-talker) as a between-participant factor and talker (novel, trained) as a within-participant factor.

\section{Results}

\section{Familiarity Judgment Task}

As expected, words were rated as more familiar $(M=2.69)$ than part-words $(M=2.53)$ and non-words $(M=2.43$; linear effect of word category: $\mathrm{B}=-0.34, \mathrm{SE}=0.07, p<.001$; Figure 2A). In the expanded model, trials from the trained talker assessment were rated as marginally more familiar compared to trials from the novel talker assessment ( $M=2.67$ vs. 2.43 , respectively; $\mathrm{B}=0.22, S E=0.13, p=.082$ ). Additionally, this effect of talker interacted with 
condition ( $\mathrm{B}=0.47, \mathrm{SE}=0.17, p=.006$ ). This interaction (Fig. $2 \mathrm{~B}$ ) is best characterized by a slight "familiar talker bias" (i.e., overall higher familiarity ratings for the trained talker) for participants in the multiple-talker condition, compared to a more pronounced "familiar talker bias" for participants in the single-talker condition. Thus, although both conditions demonstrated evidence of learning, it appears that receiving training on multiple talkers attenuates the conflation of talker familiarity and stimulus familiarity. No other term was significant in the model, including the interaction of condition and word category $(B=0.14, S E=0.21, p=.500)$ and the three-way interaction of condition, word category, and talker $(B=0.08, S E=0.29, p=.789$ ), indicating that overall rating performance was similar between the two training groups.

[[Figure 2 about here]]

\section{Forced-Choice Recognition Task}

The grand mean was $56.2 \%$, which was significantly above the chance estimate $(t(77)=$ $5.01, p<.001)$. Participants in the single-talker condition $(t(42)=2.67, p=.011)$ and the multiple-talker condition $(t(34)=4.61, p<.001)$ were both independently above chance. In the mixed-effects model, we found that participants trained with multiple talkers had overall higher accuracy compared to participants trained with a single talker ( $58.4 \%$ vs. $54.4 \%$; main effect of condition: $\mathrm{B}=-0.26, S E=0.13, p=.050$ ). We additionally observed that participants were much more likely to make a correct response when the correct answer was in the first position (60.7\%) compared to the second position (51.8\%), reflected in a significant main effect of correct answer position ( $\mathrm{B}=-0.37, \mathrm{SE}=0.08, p<.001)$. Finally, we found that participants' confidence ratings were marginally, positively associated with their overall accuracy $(B=0.12, S E=0.07, p=$ .066). No other term was significant in the model, including the interaction of condition and talker $(\mathrm{B}=0.18, \mathrm{SE}=0.16, p=.271)$.

\section{Target Detection Task}

\section{Signal Detection Analysis}

Participants in the Multiple-Talker Condition had d'values of 2.45 (SE: 0.12) and 2.35 (SE: 0.10) for the Novel and Trained Talker Tests, respectively. Participants in the Single-Talker Condition had $d$ ' values of 2.20 (SE: 0.08) and 2.30 (SE: 0.11) for the Novel and Trained Talker Tests, respectively. All of these $d$ ' values were above zero (one-sample $t$-tests against 0 : all $p$ s $<.001)$ and suggest that participants had excellent sensitivity in detecting target syllables. The main effects of Condition $(F(1,76)=1.41, p=.238)$, Test $(F(1,76)=0.00, p=.971)$, and the 
interaction of Condition and Test $(F(1,76)=2.67, p=.107)$ were all nonsignificant, indicating that target sensitivity was similar between the two training groups.

\section{Reaction Time Analysis}

Replicating prior research, we found a robust effect of syllable position on response times. This effect of syllable position had a significant linear $(B=-23.64, S E=2.61, p<.001)$ and quadratic $(B=-19.21, S E=2.40, p<.001)$ fit. This effect of syllable position is best characterized by a pronounced decrease in response times from the second to the third syllable positions, with an unanticipated, nominal increase in response times from the first to the second syllable (Fig. 3C). These significant effects of syllable position were observed even while controlling for sequence position, which was found to be significant $(B=1.42, S E=0.13, p<$ $.001)$, suggesting that participants generally responded more quickly when target syllables were presented later in the trial sequence.

In the expanded model, the quadratic fit of syllable position strongly interacted with condition $(B=24.24, S E=6.83, p<.001 ;$ Fig, 3D), suggesting that the pattern of response times across Syllable Position depended on whether participants were trained with a single talker versus multiple talkers. Specifically, we found that multiple talker training led to a stronger quadratic fit of syllable position, with slower responses to the first and second syllables and faster responses to the third syllable. Interestingly, the linear fit of syllable position also interacted with condition ( $B=13.25, S E=6.38, p=.038$ ), with multiple talker training leading to a stronger linear fit of syllable position as well.

Finally, we found a three-way interaction between the quadratic fit of syllable position, condition, and talker $(B=-21.34, S E=9.59, p=.026)$. We deconstructed this three-way interaction by creating two additional models: one modelling performance when listening to the trained talker and the other modelling performance when listening to the novel talker. The novel talker model showed a strong two-way interaction of syllable position and condition $(B=24.17$, $S E=7.02, p<.001$ ), with participants trained with multiple talkers showing a stronger quadratic decrease of response time as a function of syllable position compared to participants trained with a single talker. In contrast, the trained talker model showed no difference in the quadratic fit as a function of condition $(B=2.73, S E=6.52, p=.675)$. In other words, the significant interaction of quadratic syllable position and condition appeared to be entirely driven by the novel talker assessment. 

talker, benefits statistical learning. In particular, participants trained on multiple talkers showed better accuracy on the forced-choice recognition measure, and a stronger response-time prediction effect on the target detection task. While overall performance on the familiarity rating task was similar between conditions, participants trained with multiple talkers were better at learning studies have generally used highly controlled, invariant speech tokens.

In the forced-choice recognition task, participants were presented with two trisyllabic items on each trial and had to determine which item came from the same language as training. We observed that both groups of participants performed significantly above chance, indicating successful statistical learning. In addition, we found that multiple-talker training led to overall increased accuracy overall, across both talkers. This finding suggests that participants who were exposed to multiple talkers were better able to extract the underlying statistical regularities words. This performance difference between single- and multiple-talker participants, however, was right at our significance threshold $(p=.050)$, meaning this finding should be interpreted with caution. Overall, these findings suggest that training with multiple talkers may facilitate the explicit recognition of the underlying statistical structure of speech.

In the target detection task, participants pressed a button as quickly as possible whenever they heard a target syllable embedded within a speech stream. If participants become sensitive to the statistical structure of the speech stream, they are expected to show faster responses to more predictable syllables compared to less predictable (predictive) syllables. This response time facilitation effect can occur even without any explicit knowledge of the nonsense words, such that the target detection task is considered a more implicit measure of statistical learning (e.g., Batterink \& Paller, 2015). In line with our original prediction that multiple-talker training would facilitate statistical learning, we found that participants in the multiple-talker condition showed faster responses to third position targets. This result indicates that participants who were exposed to multiple talkers were more effective in anticipating and detecting the most predictable syllables in the continuous speech stream. 
In addition to this straightforward RT decrease (revealed by a significant linear fit), we also found an interaction with training condition and the quadratic RT fit for syllable position. This result indicates that training with multiple talkers resulted in relatively slower responses to first- and second-position syllables, along with relatively faster responses to third-position syllables regardless of test (Fig. 2C). The slower response to the initial two syllables was not predicted a priori. However, a response time delay to initial, predictive items as a function of learning has been previously observed in both the auditory (Batterink et al., 2015; Batterink, 2017) and visual (Turk-Browne et al., 2010) domains. For example, Batterink (2017) previously found that as statistical learning progresses, after just one or two exposures to a word, the responses to initial syllables slow down while the responses to third-position syllables speed up. In the visual modality, a slowdown to initial items in a sequence has been proposed to reflect implicit perceptual anticipation (Turk-Browne et al., 2010), triggered by the presence of a predictive stimulus. Another recent visual statistical learning study (Sherman \& Turk-Browne, 2020) found that when categories of scenes follow a predictive sequence, predictive scenes were less likely to be remembered than non-predictive scenes. Further, when viewing a predictive stimulus, the hippocampus begins to represent information about the upcoming scene category (Sherman \& Turk-Browne, 2020), which is associated with poorer encoding of the current item. These results suggest that there may be a neural trade-off between processing of a current item and prediction of the next. In the current study, to the extent that multiple talker training enhances statistical learning, stronger anticipatory processes may be triggered by the onset of the first two syllables in a word. Anticipatory processes to these earlier syllables may then compete with the detection and identification of the current syllable (e.g., see Turk-Browne et al., 2010), delaying RTs and producing a stronger quadratic effect. Thus, the present results are consistent with a framework in which multiple-talker training facilitates statistical learning, ultimately enhancing perceptual anticipation of the most highly predictable syllables in the sequence.

However, although prior studies using the target detection task have reported relatively more pronounced response time differences between the second and third syllables compared to the first and second (Batterink et al., 2015), the current nominal increase in response times from the first to second syllable position - observed across conditions - was unanticipated and might reflect idiosyncrasies with the particular syllable collection (cf. Siegelman, Bogaerts, Elazar, Arciuli, \& Frost, 2018). Future research should therefore assess whether this slowdown to the second syllable generalizes to different syllable collections. 
In the familiarity rating task, participants were instructed to rate the familiarity of trisyllabic speech tokens based on how much they sounded like the "language" experienced during training. Given the framing of the task, familiarity with the talker's voice should have been orthogonal to the perceived familiarity of the speech tokens. Indeed, before completing the novel talker assessment, participants were reminded that, although they had not heard this talker before, they should base their answers on how similar the sounds were to the "language" experienced during training. Despite these instructions, participants trained with a single talker were more strongly led astray by talker-voice familiarity when making their familiarity ratings. In contrast, participants who were trained with multiple talkers did not conflate stimulus familiarity with talker-voice familiarity to the same extent. These results suggest that multiple talker training reduces the likelihood of making a type of source monitoring error (Johnson, 1997); particularly, misattributing stimulus familiarity (e.g., thinking a test token sounds like a word) because of increased familiarity with the talker who produced the utterance. Specifically, switching between talkers in the multiple-talker condition might have encouraged participants to attend to higherlevel, conceptual structures within the auditory stream as opposed to the psychoacoustic properties of the talkers. In contrast, training with a single talker would not signal to participants that these different sources of information need to be separated. In other words, training with multiple talkers might provide listeners with a clearer sense of the underlying conceptual structure of the sequences, aiding in generalization to novel listening situations (Heald et al., 2017; Kleinschmidt \& Jaeger, 2015).Overall, the results from the familiarity rating task suggest that multiple talker training facilitates participants approaching the task as ideal observers (cf. Geisler \& Kersten, 2002), able to separate different sources of familiarity.

While the present results generally support our hypotheses regarding multiple-talker training, it is important to note that the relative evidence in favor of our hypotheses appears to depend on the way statistical learning is assessed. As mentioned previously, the target detection task can be considered a more implicit measure of statistical learning, whereas both the familiarity rating and forced-choice recognition tasks rely on explicit judgments of specific items based on how well they fit the learned statistical structure. Consequently, the familiarity rating and recognition task provide more opportunities for other memory and decision-making processes to bias results (e.g., conflating talker and statistical familiarity). Indeed, this appears to be a plausible explanation for the familiarity rating task, in which participants trained with a single talker tended to rate all items from this talker as more familiar than items spoken by a novel talker. 
While our first hypothesis was largely supported, we did not find any evidence in support of our second hypothesis that training with a single trained talker might lead to better statistical learning performance for that particular talker. Although participants in the single-talker training condition received four times the experience with the specific talker later used in the "trained" talker learning assessments, they did not show (1) greater differentiation of words, part-words, and non-words in the familiarity rating task, (2) higher accuracy in the forced-choice recognition task, or (3) steeper response time slopes across syllable position in the target detection task. period of exposure. This observation fits within a larger body of research demonstrating that statistical learning - at least as assessed using miniaturized artificial languages - appears to follow a logarithmic trajectory such that the strongest gains occur early in exposure (Choi et al., 2020; Siegelman, Bogaerts, Kronenfeld, et al., 2018). Indeed, prior work has found behavioral evidence of learning after just a single word presentation (Batterink, 2017) and has also reported that additional exposure (e.g., 10 minutes versus 20 minutes) does not necessarily result in improved learning (Franco et al., 2011). In sum, increased familiarity with a particular voice does not appear to produce voice-specific benefits for statistical learning, at least within the context of the current experimental paradigm.

How can this conclusion be reconciled with prior work showing evidence of improved speech understanding for familiar talkers (e.g., Johnsrude et al., 2013)? One possibility is that talker-voice familiarity has been discussed predominately in the context of challenging listening environments, such as trying to understand a familiar talker that has been energetically or informationally masked. In these listening situations, talker-voice familiarity might help listeners separate the familiar talker from competing sounds (cf. Bregman, 1990). In the present paradigm, however, all speech tokens are presented in the clear. Moreover, the present statistical learning task is more conceptual in nature, as it does not require perceptual differentiation of highly similar speech sounds. The target detection task is the most demanding of the three tasks in terms of perceptually distinguishing individual syllables; however, even in this context the to-be-differentiated syllables come from distinct categories (e.g., ba versus da versus pa). These factors may have contributed to the absence of any talker-familiarity advantages in the present study.

More generally, the present results highlight the importance of both abstract and episodic information in the statistical learning of speech. According to abstractionist theories of 
speech perception, indexical information (e.g., regarding voice identity, dialect, speaking rate) is discarded quickly after perception (Halle, 1985) in service of generating truly abstract speech categories that can generalize across listening situations (e.g., Bowers, 2000). In contrast, episodic models of speech recognition (Goldinger, 1998) reject the notion of an abstract mental lexicon, instead arguing that the "echoes" of speech persisting in long-term memory can account for effects traditionally thought to support abstractionist accounts. Recent theories have integrated both episodic and abstractionist views of speech perception into a hybrid model (Pierrehumbert, 2016); yet, it is unclear how both perspectives operate in the context of language-based statistical learning. The present results provide some clarification on this issue by suggesting that the ability to successfully generalize might depend on an interaction of indexical properties of talkers with the design of training and testing procedures. Specifically, indexical properties of speech might hinder statistical learning if they are never dissociated from the to-be-learned statistical structure of speech, as reflected by poorer performance for participants in the single-talker training condition. In contrast, indexical properties of speech might facilitate statistical learning if they can signal to listeners which sources of variability are meaningful for learning (Heald et al., 2017). In the current study, the distinctive talker properties in the multiple-talker condition might encourage listeners to attend to what is constant across talkers (i.e., the statistical structure of the syllables), facilitating learning (Vouloumanos et al., 2012).

\section{Conclusion}

The benefits of multiple-talker training have been reported in several subdomains of speech understanding, including the learning novel phonetic contrasts, (e.g., Lively et al., 1993), accent and dialect adaptation (Bradlow \& Bent, 2008; Clopper \& Pisoni, 2004), tone language word recognition (Wiener \& Lee, 2020), learning of phonotactics in infants (Seidl et al., 2014), and even phonetic training for post-lingually deaf cochlear implant users (Miller et al., 2016). Here, we extend this theme to a new aspect of speech processing - statistical learning of syllable patterns. In the present study, we found that all participants, regardless of whether they were trained on a single talker or on multiple talkers, showed clear evidence of statistical learning, suggesting that statistical learning is robust to talker variations in training. Further, we found converging evidence across multiple tasks that multiple-talker training facilitates both implicit and explicit aspects of statistical learning and allows learners more readily to disentangle talker familiarity with the familiarity of learned statistical structures. Given the putative role of statistical learning in speech segmentation and many other aspects of language 
1 acquisition (e.g., Romberg \& Saffran, 2010; Saffran \& Kirkham, 2018), these results represent

2 an important proof of principle that statistical learning continues to operate-and may actually

3 be enhanced - with increased acoustic variability in the signal. These findings add to growing

4 evidence for the applicability of statistical learning outside of experimental contexts (i.e., in "real"

5 language acquisition contexts).

6 


\section{References}

Altenberg, E. P. (2005). The perception of word boundaries in a second language. Second Language Research, 21(4), 325-358.

Amitay, S., Hawkey, D. J. C., \& Moore, D. R. (2005). Auditory frequency discrimination learning is affected by stimulus variability. Perception and Psychophysics, 67(4), 691-698. https://doi.org/10.3758/BF03193525

Aslin, R. N., Saffran, J. R., \& Newport, E. L. (1998). Computation of Conditional Probability Statistics by 8-Month-Old Infants. Psychological Science, 9(4), 321-324.

Baayen, R. H., Davidson, D. J., \& Bates, D. M. (2008). Mixed-effects modeling with crossed random effects for subjects and items. Journal of Memory and Language, 59(4), 390-412. https://doi.org/10.1016/j.jml.2007.12.005

Bates, D., Maechler, M., Bolker, B., Walker, S., Haubo, R., Christensen, B., Singmann, H., Dai, B., Scheipl, F., Grothendieck, G., Green, P., \& Fox, J. (2020). Package “Ime4” (Lme4.rForge.r-Project.Org). https://github.com//me4/Ime4/

Batterink, L. J. (2017). Rapid Statistical Learning Supporting Word Extraction From Continuous Speech. Psychological Science, 28(7), 921-928. https://doi.org/10.1177/0956797617698226

Batterink, L. J., \& Paller, K. A. (2017). Online neural monitoring of statistical learning. Cortex, 90, 31-45. https://doi.org/10.1016/j.cortex.2017.02.004

Batterink, L. J., \& Paller, K. A. (2019). Statistical learning of speech regularities can occur outside the focus of attention. Cortex, 115, 56-71. https://doi.org/10.2307/j.ctt1df4h3s.44

Batterink, L. J., Reber, P. J., Neville, H. J., \& Paller, K. A. (2015). Implicit and explicit contributions to statistical learning. Journal of Memory And, 83, 62-78. https://doi.org/10.1016/j.jml.2015.04.004

Batterink, L. J., Reber, P. J., \& Paller, K. A. (2015). Functional differences between statistical learning with and without explicit training. Learning \& Memory, 22, 544-556.

Bosker, H. R., \& Reinisch, E. (2017). Foreign Languages Sound Fast : Evidence from Implicit Rate Normalization. Frontiers in Psychology, 8(1063), 1-13. https://doi.org/10.3389/fpsyg.2017.01063 
Bowers, J. S. (2000). In defense of abstractionist theories of repetition priming and word identification. Psychonomic Bulletin and Review, 7(1), 83-99. https://doi.org/10.3758/BF03210726

Bradlow, A. R., \& Bent, T. (2008). Perceptual adaptation to non-native speech. Cognition, 106(2), 707-729. https://doi.org/10.1016/j.cognition.2007.04.005

Choi, D., Batterink, L. J., Black, A. K., Paller, K. A., \& Werker, J. F. (2020). Preverbal Infants Discover Statistical Word Patterns at Similar Rates as Adults: Evidence From Neural Entrainment. Psychological Science, 31(9), 1161-1173. https://doi.org/10.1177/0956797620933237

Christensen, R. H. B. (2019). A Tutorial on fitting Cumulative Link Mixed Models with clmm2 from the ordinal Package (Vol. 1, Issue 1).

Clopper, C. G., \& Pisoni, D. B. (2004). Effects of Talker Variability on Perceptual Learning of Dialects. 3.

Cole, R. A., \& Jakimik, J. (1980). How are syllables used to recognize words? The Journal of the Acoustical Society of America, 67(3), 965-970. https://doi.org/10.1121/1.383939

Domingo, Y., Holmes, E., Macpherson, E., \& Johnsrude, I. S. (2019). Using spatial release from masking to estimate the magnitude of the familiar-voice intelligibility benefit. The Journal of the Acoustical Society of America, 146(5), 3487-3494. https://doi.org/10.1121/1.5133628

Ettlinger, M., Morgan-Short, K., Faretta-Stutenberg, M., \& Wong, P. C. M. (2016). The Relationship Between Artificial and Second Language Learning. Cognitive Science, 40, 822-847. https://doi.org/10.1111/cogs.12257

Evans, J. L., Saffran, J. R., \& Robe-Torres, K. (2009). Statistical Learning in Children with Specific Language Impairment. Journal of Speech, Language, and Hearing Research, 52, 321-335.

Franco, A., Cleeremans, A., \& Destrebecqz, A. (2011). Statistical learning of two artificial languages presented successively: How conscious? Frontiers in Psychology, 2(SEP), 112. https://doi.org/10.3389/fpsyg.2011.00229

Frost, R. L. A., Jessop, A., Durrant, S., Peter, M. S., Bidgood, A., Pine, J. M., Rowland, C. F., \& Monaghan, P. (2020). Non-adjacent dependency learning in infancy, and its link to language development. Cognitive Psychology, 120(July 2019), 101291. 
https://doi.org/10.1016/j.cogpsych.2020.101291

Geisler, W. S., \& Kersten, D. (2002). Illusions, perception and Bayes. Nature Neuroscience, 5(6), 508-510. https://doi.org/10.1038/nn0602-508

Goldinger, S. D. (1998). Echoes of Echoes? An Episodic Theory of Lexical Access. 105(2), 251-279.

Graf Estes, K., Evans, J. L., Alibali, M. W., \& Saffran, J. R. (2007). Can Infants Map Meaning to Newly Segmented Words? Statistical Segmentation and Word Learning. Psychological Science, 18(3), 254-260.

Halle, M. (1985). Speculations about the representation of words in memory. In V. A. Fromkin (Ed.), Phonetic Linguistics. Academic Press.

Hauser, M. D., Newport, E. L., \& Aslin, R. N. (2001). Segmentation of the speech stream in a non- human primate: statistical learning in cotton-top tamarins. Cognition, 78, 53-64.

Heald, S. L. M., Van Hedger, S. C., \& Nusbaum, H. C. (2017). Perceptual plasticity for auditory object recognition. Frontiers in Psychology, 8(MAY). https://doi.org/10.3389/fpsyg.2017.00781

Holmes, E., Domingo, Y., \& Johnsrude, I. S. (2018). Familiar Voices Are More Intelligible, Even if They Are Not Recognized as Familiar. Psychological Science, 29(10), 1575-1583. https://doi.org/10.1177/0956797618779083

Johnson, E. K., \& Jusczyk, P. W. (2001). Word Segmentation by 8-Month-Olds: When Speech Cues Count More Than Statistics. Journal of Memory and Language, 44, 548-567. https://doi.org/10.1006/jmla.2000.2755

Johnson, M. K. (1997). Source monitoring and memory distortion. Philosophical Transactions of the Royal Society B: Biological Sciences, 352(1362), 1733-1745. https://doi.org/10.1098/rstb.1997.0156

Johnsrude, I. S., Mackey, A., Hakyemez, H., Alexander, E., Trang, H. P., \& Carlyon, R. P. (2013). Swinging at a Cocktail Party: Voice Familiarity Aids Speech Perception in the Presence of a Competing Voice. Psychological Science, 24(10), 1995-2004. https://doi.org/10.1177/0956797613482467

Jusczyk, P. W. (1999). How infants begin to extract words from speech. Trends in Cognitive 
Sciences, 3(9), 323-328.

Karni, A., \& Sagi, D. (1991). Where practice makes perfect in texture discrimination: Evidence for primary visual cortex plasticity. Proceedings of the National Academy of Sciences of the United States of America, 88(11), 4966-4970. https://doi.org/10.1073/pnas.88.11.4966

Kittleson, M. M., Aguilar, J. M., Tokerud, G. L., Plante, E., \& Asbøjrnsen, A. E. (2010). Implicit language learning: Adults' ability to segment words in Norwegian. Bilingualism, 13(4), 513523. https://doi.org/10.1017/S1366728910000039

Kleinschmidt, D. F., \& Florian Jaeger, T. (2015). Robust speech perception: Recognize the familiar, generalize to the similar, and adapt to the novel. Psychological Review, 122(2), 148-203. https://doi.org/10.1037/a0038695

Lany, J., Shoaib, A., Thompson, A., \& Estes, K. G. (2018). Infant statistical-learning ability is related to real-Time language processing. Journal of Child Language, 45(2), 368-391. https://doi.org/10.1017/S0305000917000253

Leeuw, J. R. De. (2015). jsPsych : A JavaScript library for creating behavioral experiments in a Web browser. Behavioral Research, 47(1), 1-12. https://doi.org/10.3758/s13428-014-0458y

Litman, L., Robinson, J., \& Abberbock, T. (2017). TurkPrime.com : A versatile crowdsourcing data acquisition platform for the behavioral sciences. Behavior Research Methods, 49, 433-442. https://doi.org/10.3758/s13428-016-0727-z

Lively, S. E., Logan, J. S., \& Pisoni, D. B. (1993). Training Japanese listeners to identify English $/ \mathrm{r} /$ and $/ \mathrm{I} /$. II: The role of phonetic environment and talker variability in learning new perceptual categories. Journal of the Acoustical Society of America, 94(3), 1242-1255. https://doi.org/10.1121/1.408177

Macmillan, N. A., \& Creelman, C. D. (2005). Classification Designs for Discrimination. In Detection Theory: A User's Guide. https://doi.org/10.1017/CBO9781107415324.004

Magnuson, J. S., \& Nusbaum, H. C. (2007). Acoustic Differences, Listener Expectations, and the Perceptual Accommodation of Talker Variability. Journal of Experimental Psychology: Human Perception and Performance, 33(2), 391-409.

Magnuson, J. S., Yamada, R. A., \& Nusbaum, H. C. (1994). Are representations used for talker udentification available for talker normalization? Proceedings of the International 
Conference of Speech and Language Processing, 1-5.

Miller, S. E., Zhang, Y., \& Nelson, P. (2016). Efficacy of Multiple-Talker Phonetic Identification Training in Postlingually Deafened Cochlear Implant Listeners. Journal of Speech, Language, and Hearing Research, 59, 90-98.

Misyak, J. B., \& Christiansen, M. H. (2012a). Statistical Learning and Language: An Individual Differences Study. Language Learning, 62(1), 302-331. https://doi.org/10.1111/j.14679922.2010.00626.x

Misyak, J. B., \& Christiansen, M. H. (2012b). Statistical Learning and Language: An Individual Differences Study. Language Learning, 62(1), 302-331. https://doi.org/10.1111/j.14679922.2010.00626.x

Misyak, J. B., Christiansen, M. H., \& Tomblin, J. B. (2010). On-line individual differences in statistical learning predict language processing. Frontiers in Psychology, 1(SEP), 1-9. https://doi.org/10.3389/fpsyg.2010.00031

Mullennix, J. W., Pisoni, D. B., \& Martin, C. S. (1989). Some effects of talker variability on spoken word recognition. The Journal of the Acoustical Society of America, 85(1), 365378.

Newport, E. L., \& Aslin, R. N. (2004). Learning at a distance I. Statistical learning of nonadjacent dependencies. Cognitive Psychology, 48, 127-162. https://doi.org/10.1016/S0010-0285(03)00128-2

Nusbaum, H., \& Magnuson, J. (1997). Talker Normalization : Phonetic Constancy as a Cognitive Process. Talker Variability and Speech Processing, 109-132. https://doi.org/10.1121/1.2028337

Nygaard, L. C., \& Pisoni, D. B. (1998). Talker-specific learning in speech perception. Perception and Psychophysics, 60(3), 355-376. https://doi.org/10.3758/BF03206860

Pelucchi, B., Hay, J. F., \& Saffran, J. R. (2009). Statistical Learning in a Natural Language by 8Month-Old Infants. 80(3), 674-685.

Peterson, G. E., \& Barney, H. L. (1952). Control Methods Used in a Study of the Vowels variation is to be found in the. Journal of the Acoustical Society of America, 175(2), 175184. https://doi.org/10.1121/1.1906875 
Pierrehumbert, J. B. (2016). Phonological Representation: Beyond Abstract Versus Episodic. Annual Review of Linguistics, 2(1). https://doi.org/10.1146/annurev-linguist-030514-125050

Plante, E., Patterson, D., Gómez, R., Almryde, K. R., White, M. G., \& Asbjørnsen, A. E. (2015). The nature of the language input affects brain activation during learning from a natural language. Journal of Neurolinguistics, 36, 17-34. https://doi.org/10.1016/j.jneuroling.2015.04.005

Posner, M. I., \& Keele, S. W. (1968). On the genesis of abstract ideas. Journal of Experimental Psychology, 77(3), 353-363.

Romberg, A. R., \& Saffran, J. R. (2010). Statistical learning and language acquisition. Wiley Interdisciplinary Reviews: Cognitive Science, 1(6), 906-914. https://doi.org/10.1002/wcs.78

Saffran, J. R., Aslin, R. N., \& Newport, E. L. (1996). Statistical Learning by 8-Month-Old Infants. Science, 274(5294), 1926-1928.

Saffran, J. R., Newport, E. L., Aslin, R. N., Tunick, R. A., \& Barrueco, S. (1997). Incidental language learning: Listening (and Learning) out of the Corner of Your Ear. Psychological Science, 8(2), 101-105.

Schwab, J. F., Schuler, K. D., Stillman, C. M., Newport, E. L., Jr, J. H. H., \& Howard, D. V. (2016). Aging and the Statistical Learning of Grammatical Form Classes. Psychology of Aging, 31(5), 481-487.

Seidl, A., Onishi, K. H., \& Cristia, A. (2014). Talker Variation Aids Young Infants' Phonotactic Learning. Language Learning and Development, 10(4), 297-307. https://doi.org/10.1080/15475441.2013.858575

Siegelman, N. (2020). Statistical learning abilities and their relation to language. Language and Linguistics Compass, 14(3), 1-19. https://doi.org/10.1111/lnc3.12365

Siegelman, N., Bogaerts, L., Elazar, A., Arciuli, J., \& Frost, R. (2018). Linguistic entrenchment : Prior knowledge impacts statistical learning performance. Cognition, 177(August 2017), 198-213. https://doi.org/10.1016/j.cognition.2018.04.011

Siegelman, N., Bogaerts, L., Kronenfeld, O., \& Frost, R. (2018). Redefining "Learning" in Statistical Learning: What Does an Online Measure Reveal About the Assimilation of Visual Regularities? Cognitive Science, 42(1996), 692-727. https://doi.org/10.1111/cogs.12556 


\section{9}

Toro, J. M., Sinnett, S., \& Soto-Faraco, S. (2005). Speech segmentation by statistical learning depends on attention. Cognition, 97, 25-34. https://doi.org/10.1016/j.cognition.2005.01.006

Toro, J. M., \& Trobalón, J. B. (2005). Statistical computations over a speech stream in a rodent. $67(5), 867-875$.

Tussing, A. A., \& Greene, R. L. (2001). Effects of Familiarity Level and Repetition on Recognition Accuracy. The American Journal of Psychology, 114(1), 31-41.

Vouloumanos, A., Brosseau-liard, P. E., Balaban, E., Hager, A. D., Monaghan, P., \& Bonatti, L. L. (2012). Are the products of statistical learning abstract or stimulus-specific? 3(March), 111. https://doi.org/10.3389/fpsyg.2012.00070

Wiener, S., \& Lee, C. Y. (2020). Multi-Talker Speech Promotes Greater Knowledge-Based Spoken Mandarin Word Recognition in First and Second Language Listeners. Frontiers in Psychology, 11(February), 1-14. https://doi.org/10.3389/fpsyg.2020.00214

Wong, P. C. M., Nusbaum, H. C., \& Small, S. L. (2004). Neural Bases of Talker Normalization. Journal of Cognitive Neuroscience, 16(7), 1-13. https://doi.org/10.1162/0898929041920522

Wright, B. A., Buonomano, D. V., Mahncke, H. W., \& Merzenich, M. M. (1997). Learning and generalization of auditory temporal-interval discrimination in humans. Journal of Neuroscience, 17(10), 3956-3963. https://doi.org/10.1523/jneurosci.17-10-03956.1997 


\section{Figures}

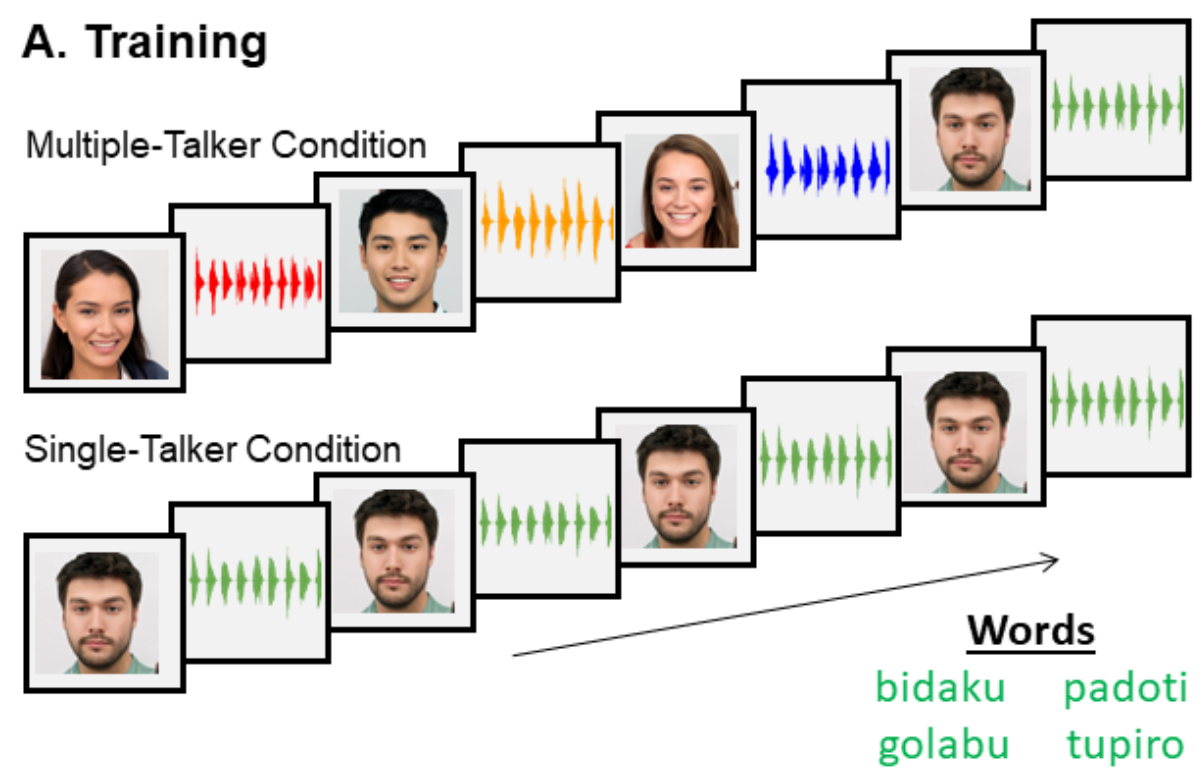

B. Target Familiarity Task

$\left.\begin{array}{l}\text { golabu } \\ \text { golati } \\ \text { godapi }\end{array}\right\} \begin{array}{lllll}1 & 2 & 3 & 4 & \frac{12 \text { word }}{4 \text { Familiarity }} \\ \text { Famord }\end{array}$

\section{Forced-Choice Recognition Task

$\begin{array}{ccccccc}1 & 2 & 1 & 2\end{array} \quad \begin{array}{cccc}1 & 2 & 3 & 8 \text { correct }=1 \\ \text { bidaku } & \text {.. robiti }\end{array} \rightarrow$ Correct $? \rightarrow \begin{gathered}\text { Confidence } \\ 8 \text { correct }=2\end{gathered}$

\section{Target Detection Task}

sample third syllable stream

$$
\text { Listen for } \boldsymbol{k} \boldsymbol{u} \rightarrow \underbrace{\text { tupirobidakugolabubidaku... }}_{16 \text { words (48 syllables) }}
$$

\section{4 streams}

8 first syllable

8 second syllable

8 third syllable

4 targets per stream

Figure 1: Overview of experimental paradigm. Panel A represents the training component of the experiment for the multiple-talker (top) and single-talker (bottom) conditions. Each condition contained four listening blocks of 540 syllables each. Panel B depicts the Familiarity Rating Task. Panel $C$ depicts the Forced-Choice Recognition Task. Panel D represents the Target Detection Task. In the case of the multiple-talker condition, the designated trained talker was the final talker presented during training. Test order was counterbalanced, with participants either receiving the novel talker assessment first or the trained talker assessment first. 
1
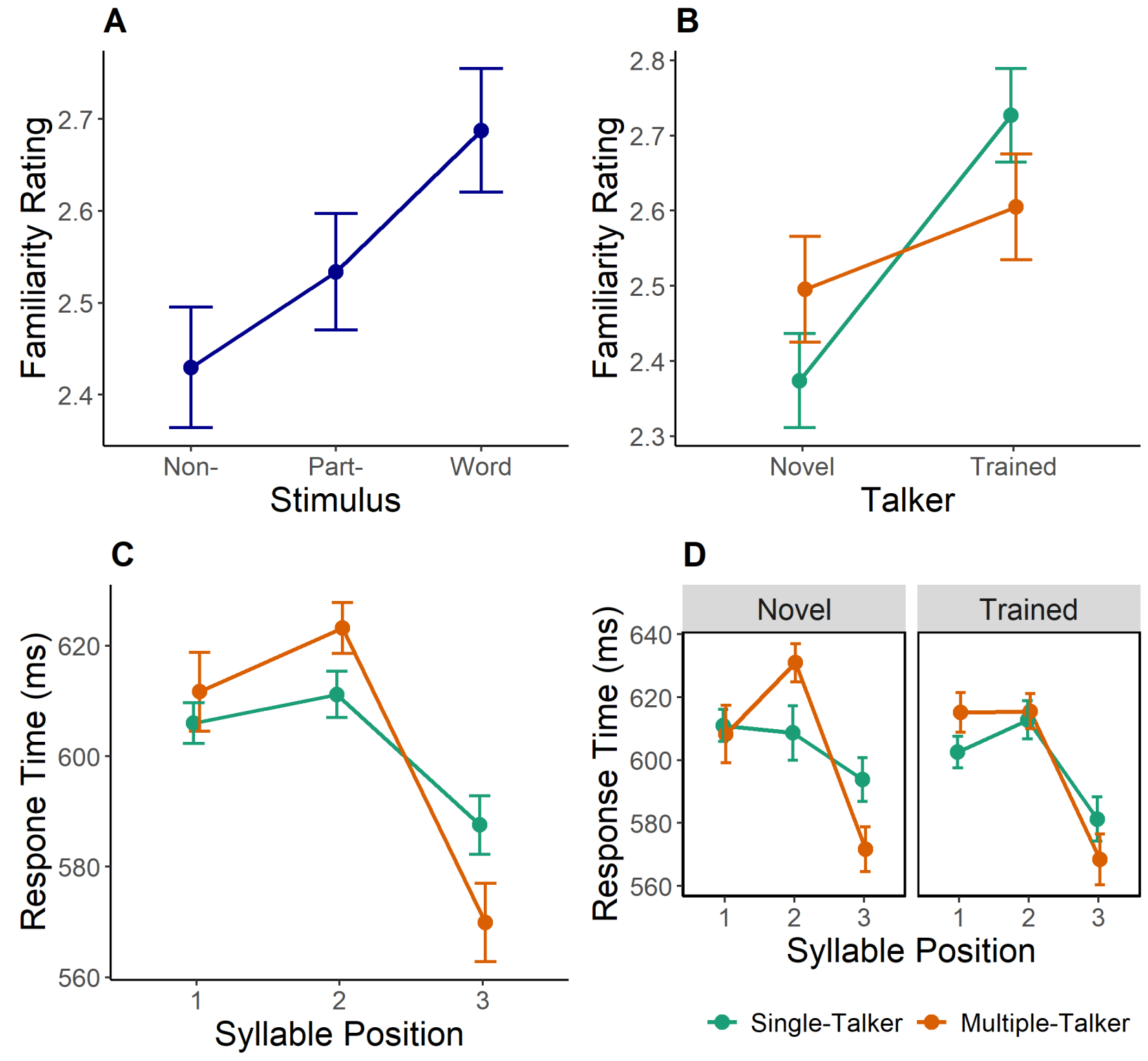

Figure 2: Summarized results from the statistical learning assessments. Panel $A$ depicts ratings for nonwords, part-words, and words in the Familiarity Rating Task. Panel B depicts the interaction of condition and talker in the Familiarity Rating Task. Panel $C$ depicts mean response times across syllable position and condition for the Target Detection Task. Panel D depicts the three-way interaction of syllable position, condition, and talker in the Target Detection Task. Error bars represent \pm 1 standard error of the mean. 